\title{
INFINITESIMAL DEFORMATION OF THE SKEW HELICOID.
}

\author{
BY DR. L. P. EISENHART.
}

(Read before the American Mathematical Society, September 3, 1902.)

Consider the skew helicoid $S$, defined by the equations

$$
x=u \cos v, \quad y=u \sin v, \quad z=a v .
$$

We shall show that the problem of the infinitesimal deformation of this surface can be completely solved.

By direct calculation we find

$$
\begin{gathered}
E=\sum\left(\frac{\partial x}{\partial u}\right)^{2}=1, \quad F=\sum \frac{\partial x}{\partial u} \frac{\partial x}{\partial v}=0 \\
G=\sum\left(\frac{\partial x}{\partial v}\right)^{2}=u^{2}+a^{2}
\end{gathered}
$$

and

$$
X, Y, Z=\frac{a \sin v,-a \cos v, u}{\sqrt{u^{2}+a^{2}}}
$$

where $Y, X, Z$ denote the direction cosines of the normal. Again we find

$$
D=\sum X \frac{\partial^{2} x}{\partial u^{2}}=0, \quad D^{\prime}=\sum X \frac{\partial^{2} x}{\partial u \partial v}=\frac{-a}{\sqrt{u^{2}+a^{2}}},
$$

$$
D^{\prime \prime}=\sum X \frac{\partial^{2} x}{\partial v^{2}}=0
$$

The characteristic equation of the deformation reduces in this case to

$$
\frac{\partial^{2} \phi}{\partial u \partial v}+\frac{u}{u^{2}+a^{2}} \frac{\partial \phi}{\partial v}=0
$$

of which the general integral is

$$
\phi=\frac{U+V}{\sqrt{u^{2}+a^{2}}}
$$


where $U$ is a function of $u$ alone and $V$ is a function of $v$ alone.

The cartesian cöordinates of the surface $S_{1}$, corresponding to $S$ with orthogonality of linear elements, have the following expressions : *

$$
\begin{aligned}
& x_{1}=(U+V) \sin v-2 \int \sin v \cdot V^{\prime} d v, \\
& y_{1}=-(U+V) \cos v+2 \int \cos v \cdot V^{\prime} d v, \\
& z_{1}=-\frac{1}{a}\left[(U+V) u-2 \int u \cdot U^{\prime} d u\right],
\end{aligned}
$$

where the accent denotes differentiation. From (6) we have that, when $V$ is a constant, $S_{1}$ is a surface of revolution. Moreover, since these formulæ involve an arbitrary function of $u$, it follows that any surface of revolution can be defined by them.

Conversely, given a surface of revolution defined by

$$
x=u \cos v, \quad y=u \sin v, \quad z=U ;
$$

the helicoid with plane director, whose equations are

$$
\bar{x}=U_{1} \sin v, \quad \bar{y}=-U_{1} \cos v, \quad \bar{z}=\alpha v,
$$

has the same axis and corresponds with orthogonality of linear elements, if

$$
U_{1}=a u \int \frac{U^{\prime}}{u^{2}} d u
$$

where the accent denotes differentiation with respect to $u$.

By direct calculation we find from (6),

$$
F_{1}=\sum \frac{\partial x_{1}}{\partial u} \frac{\partial x_{1}}{\partial v}=\frac{V^{\prime}}{a^{2}}\left[u(U+V)-\left(u^{2}+a^{2}\right) U^{\prime}\right]
$$

From (4) we see that the lines $u=$ const., $v=$ const. on $S$ are asymptotic, and consequently the corresponding lines on $S_{1}$ form a conjugate system. Hence it follows from (7) that the necessary and sufficient condition that asymptotic lines on S cor-

* Bianchi, Lezioni, p. 276. 
respond to lines of curvature on $S_{1}$ is that $V^{\prime}=0$, that is, $S_{1}$ must be a surface of revolution.

From (5) we see that in the latter case $\phi$ is a function of $u$ alone. This, however, is a general property of the infinitesimal deformation of minimal surfaces. For, from the following formula, which we have established elsewhere,*

$$
F_{1}=F \phi^{2}+\frac{1}{K^{2}\left(E G-F^{2}\right)}\left(D \frac{\partial \phi}{\partial v}-D^{\prime} \frac{\partial \phi}{\partial u}\right)\left(D^{\prime} \frac{\partial \phi}{\partial v}-D^{\prime \prime} \frac{\partial \phi}{\partial u}\right),
$$

it is seen that when $S$ is a minimal surface referred to its asymptotic lines, the necessary and sufficient condition that the parametric lines on $S_{1}$ be the lines of curvature is that $\phi$ shall be a function of $u$ alone or a function of $v$ alone.

When in particular we take

$$
U=\sqrt{u^{2}+a^{2}}, \quad V=0,
$$

we get

$$
\begin{gathered}
x_{1}=\sqrt{u^{2}+a^{2}} \cdot \sin v, \quad y_{1}=-\sqrt{u^{2}+a^{2}} \cdot \cos v, \\
z_{1}=-a \log \left(u+\sqrt{u^{2}+a^{2}}\right),
\end{gathered}
$$

which define the catenoid. From (5) we get $\phi=1$, which is the case whenever, in the deformation of a minimal surface, the adjoint of the latter is taken for the surface $S_{1} . \dagger$

Genty \& has shown that the cartesian coördinates, $x_{0}, y_{0}, z_{0}$, of the associate surface $\S S_{0}$ in an infinitesimal deformation are given by the equations

$$
\begin{aligned}
& d x_{1}=z_{0} d y-y_{0} d z \\
& d y_{1}=x_{0} d z-z_{0} d x \\
& d z_{1}=y_{0} d x-x_{0} d y
\end{aligned}
$$

Substituting the expressions for $x, y, \cdots, z_{1}$, from (1) and (6), and solving we find

$$
\begin{aligned}
& x_{0}=\frac{1}{a}\left[\left(U+V-u U^{\prime}\right) \sin v+V^{\prime} \cdot \cos v\right], \\
& y_{0}=\frac{1}{a}\left[-\left(U+V-u U^{\prime}\right) \cos v+V^{\prime} \cdot \sin v\right], \\
& z_{0}=U^{\prime} .
\end{aligned}
$$

* Amer. Jour. of Math., vol. 24, p. 177.

$\dagger$ lbid., p. 192.

$\ddagger$ Toulouse Annales, vol. 9.

\& Bianchi, l. c., p. 279 . 
The linear element of $S_{0}^{r}$ is readily found to be

$$
d s_{0}{ }^{2}=\frac{U^{\prime 2}}{a^{2}}\left(u^{2}+a^{2}\right) d u^{2}+\frac{1}{a^{2}}\left(V^{\prime \prime}+V-u U^{\prime}+U\right)^{2} d v^{2}
$$

It is well known that the lines upon any associate surface corresponding to the asymptotic lines on $S$ form a conjugate system. From (10) we see that the conjugate system on $S_{0}$ corresponding to asymptotic lines on $S$ are the lines of curvature. Furthermore, the lines of curvature $v=$ const. are geodesics and consequently $S_{0}$ is a surface of Monge.* From the form of the coefficient of $d v^{2}$ in (10) we have that the generating developable is a cylinder. $\dagger$ Hence

In any infinitesimal deformation of a skew helicoid the associate surface is a moulure surface.

Conversely, given any moulure surface; its equations can be put in the form (9) and then can be taken for the associate surface in the deformation of the helicoid (1), corresponding to the value $(U+V)\left(u^{2}+a^{2}\right)^{-1 / 2}$ of the characteristic function.

From (6) and (9) we get the

THEOREM: When the surface $S_{1}$ in an infinitesimal deformation of a skew helicoid is a surface of revolution, the associate surface $S_{0}$ also is a surface of revolution, and their lines of curvature correspond.

And conversely,

When the associate surface $S_{0}$ is a surface of revolution, the characteristic surface $S_{1}$ is a surface of revolution.

When in particular $S_{1}$ is the catenoid, $S_{0}$ is the sphere of radius unity and center at the origin.

If we put

$$
U=a \sqrt{u^{2}+a^{2}}-a u \log \left(u+\sqrt{u^{2}+a^{2}}\right), \quad V=0,
$$

the formulæ (9) define the catenoid. We have shown that the necessary and sufficient condition that the lines of curvature be unaltered in the deformation of $S$ is that $S_{0}$ be the adjoint minimal surface of $S$. Hence, when

$$
\phi=a\left[1-\frac{u}{\sqrt{u^{2}+a^{2}}} \log \left(u+\sqrt{u^{2}+a^{2}}\right)\right],
$$

* Monge, Applic. de l'Analyse a la Géométrie 5 ed., chap. 25.

$\dagger$ Darboux, Leçons, vol. 1, p. 105.

†. c.. p. 199 . 
the corresponding deformation of $S$ leaves the lines of curvature unaltered and only in this case.

Princeton,

June, 1902 .

\section{ON INTEGRABILITY BY QUADRATURES.}

BY DR. SAUL EPSTEEN.

(Read before the American Mathematical Society, September 3, 1902.)

THE object of this note is to show that Vessiot's noted theorem that: " the necessary and sufficient condition that a linear differential equation shall be integrable by quadratures is that its group of rationality shall be integrable," * is a special case of the Jordan-Beke $\uparrow$ theorem on reducibility of differential equations.

The Jordan-Beke theorem is to the effect that "if a linear differential equation is reducible in the sense of Frobenius $f$ then its group of rationality will transform a certain linear manifoldness of the solutions (which does not include the total $n$-dimensional manifoldness) into itself."

Analytically interpreted $\S$ this says that the group

(1)

$$
\begin{aligned}
& y_{1}=a_{11} y_{1}+\cdots+a_{1 k} y_{k v}, \\
& y_{k}=a_{k 1} y_{1}+\cdots+a_{k k} y_{k},
\end{aligned}
$$

$$
\begin{aligned}
& y_{k+1}=a_{k+1,1} y_{1}+\cdots+a_{k+1, k} y_{k}+a_{k+1, k+1} y_{k+1}+\cdots+a_{k+1, n} y_{n}, \\
& y_{n}=a_{n 1} y_{1}+\cdots+a_{n k} y_{k}+a_{n, k+1} y_{k+1}+\cdots+a_{n n} y_{n},
\end{aligned}
$$

is isomorphic with the group of rationality. For convenience it is well to adopt Loewy's notation, writing for (1) simply the coefficients

* Vessiot : Ann. de l'Ec. nor. sup., 1892.

†C. Jordan. Bull. de la Soc. Math. de France, vol. 2 ; Beke : Math. Annalen, vol. 45 , p. 279 .

†Frohenius: Crelle, vol. 76.

\%. A. Loewy : "Ueber die irreduciblen Factoren," etc., Berichte der math.phy. Classe der Königl. Sächs. Gesellschaft der Wissenschaften zu Leipzig, vol. 54 (1902), pp. 1-13. 